American Journal of Pharmacology and Toxicology 3 (1): 59-71, 2008

ISSN 1557-4962

(C) 2008 Science Publications

\title{
Hormesis: Principles and Applications for Pharmacology and Toxicology
}

\author{
Edward J. Calabrese \\ Department of Public Health, Environmental Health Sciences, Morrill I, N344, \\ University of Massachusetts Amherst, MA 01003
}

\begin{abstract}
The present assessment places hormetic dose responses within an historical context, documenting its origin, rejection by the biomedical community during the early decades of the $20^{\text {th }}$ due to its close association with homeopathy and its revitalization during the later decades of the past century as the biomedical and toxicological communities became very interested in low dose effects of drugs and environmental agents. Particular attention is directed to assessing the quantitative features of the hormetic dose response, its underlying mechanisms and its implications for drug development and environmental risk assessment.
\end{abstract}

Key words: Hormesis, biphasic, U-shaped, J-shaped, adaptive response, drug discovery, drug development,

\section{INTRODUCTION}

Biphasic dose response relationships are commonly observed in the toxicological and pharmacological literature. Such dose response relationships have been typically observed as being a low dose stimulation while inhibition occurs at higher doses. These dose responses have generated a broad spectrum of descriptive terms that are often discipline specific. For example, biphasic dose responses have been referred to as bimodal, bidirectional, bell-shaped, bitonic, Ushaped, inverted U-shaped, J-shaped, non-monotonic, hormetic, functional antagonism, Hueppe's Rule, Arndt-Schulz Law, Yerkes-Dodson Law and overcompensation stimulation, amongst others. Since the descriptors employed are diverse, yet often with a distinct discipline-specific orientation, it has been difficult to compare the broad spectrum of biphasic dose responses against similar and objective criteria. Nonetheless, systematic attempts over the past decade to provide comparative assessments of the quantitative features of such interdisciplinary biphasic dose response relationships have revealed remarkable similarities with respect to their maximum stimulatory response, width of the stimulation, relationship of the stimulation to the toxic or pharmacological threshold, temporal context and mechanistic strategies to achieve the biphasic dose response ${ }^{[1]}$.

These observations raise numerous questions, including what is the frequency of biphasic dose responses in the toxicological and pharmacological literature; how does its frequency compare with that of other dose response models, such as the threshold or linear at low dose models; is there a dose response model that is most common and fundamental within the biological/biomedical sciences or are they unique for different endpoints? Are there quantitative features of the biphasic dose response that are common across biological systems, endpoints and chemical classes; if so, then what would be the selective advantage of such a strategy and what would be its biomedical implications; how have pharmaceutical companies exploited knowledge of dose response relationships, especially biphasic characteristics in designing, testing and optimizing drug treatments? How have biphasic dose responses affected strategies of environmental regulatory agencies?

In this article the concept of hormetic dose responses will be introduced. It will address its historical foundations, the generalizability of hormetic dose responses, their quantitative features, the implications of hormetic dose responses for biological model selection, study design and data interpretation, mechanistic foundations and its implications for pharmacology, toxicology and the biomedical sciences, in general.

\section{WHAT IS HORMESIS?}

Hormesis is a dose-response relationship that is characterized by a low dose stimulation and a high dose inhibition. The dose response relationship may be graphed to illustrate an inverted-U shaped dose response or a J-shaped dose-response depending on the endpoint being assessed (Fig. 1). In the cases of memory, longevity and growth the dose responses are 
Am. J. Pharm. \& Toxicol., 3 (1): 59-71, 2008

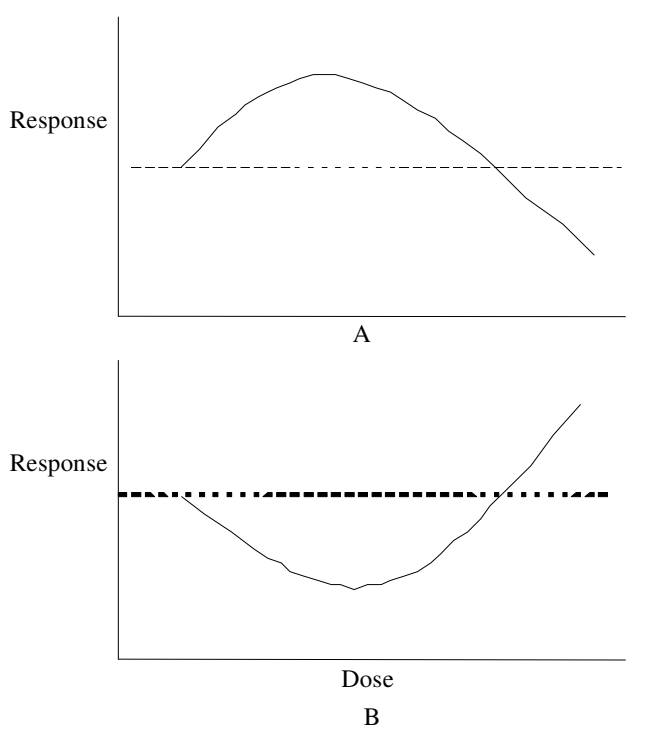

Fig. 1: General forms of the hormetic dose-response relationship (A) The most common form of the hormetic dose-response curve depicting lowdose stimiulatory and high-dose inhibitory responses, the $\beta$-or inverted U-shaped curve. (B) The hormetic dose-response curve depicting low-dose reduction and high-dose enhancement of adverse effects, the J-or-Ushaped curve

typically graphed as an inverted-U shaped dose response. If various types of disease incidence were being plotted, such as cancer incidence, the graph would appear as a J-shape. In the toxicological domain, the low dose stimulatory response may result from an overcompensation to a disruption in homeostasis. In this instance, the hormetic stimulation is an adaptive response that is reparative in nature. The low dose stimulation occurs as a result of the reparative process slightly to moderately (i.e., percentage rather than fold increases) exceeding control values. The overcompensation response is modest, thereby representing an efficient use of biological resources that assures that the repair process is completed in a timely fashion. In addition to repairing the damaged system, the low dose response also induces protection against the harmful effects of more massive subsequent exposures. The low dose adaptive response is very generalizable, affecting most, if not all, biological systems. Similar to that described above, the adapting dose also demonstrates the inverted U-shaped dose response, reflecting a system of biological optimality. The fact that the modest overcompensation response can protect against often lethal subsequent exposure
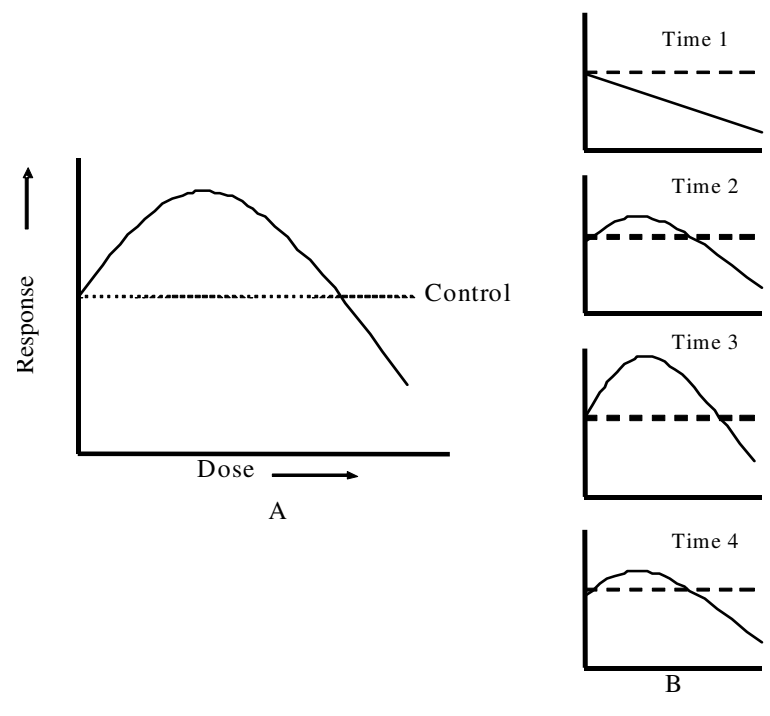

Fig. 2: (A) The general form of hormetic dose-response relationships; (B) the temporal sequence of hormetic dose-response relationship

suggests that this process reflects an efficient integrative leveraging of adaptive processes.

Since the hormetic dose response represents an overcompensation to induced damage, this is best studied within the context of a dose-time response framework. However, most investigations of hormetic dose responses do not incorporate a temporal component, typically selecting multiple doses but only a single time point for evaluation. As seen in Fig. 2, exposure to a toxic agent may initially induce a dose dependent toxic response; overtime, the biological system develops a response that not only repairs the initial damage but also results in the modest overcompensation response. The use of multiple repeat sampling or temporal measures provides the opportunity to gain considerable insights to the nature of the dose response.

Biphasic dose response relationships, which have similar quantitative features to that of the overcompensation response noted above, can occur as a direct stimulation. These often occur via the involvement of pharmacological receptor systems. These types of dose responses clearly act via different mechanisms than for the overcompensation stimulation. However, in practice they are not readily distinguishable from the overcompensation method since about $75 \%$ of the studies in the hormesis data base have utilized only a single time point ${ }^{[1]}$. Nonetheless, there are enough cases in the database to clearly distinguish direct versus overcompensation stimulation when the study designs are sufficiently strong. 
Am. J. Pharm. \& Toxicol., 3 (1): 59-71, 2008

\section{HISTORICAL FOUNDATIONS}

The idea that agents acting upon biological systems induce biphasic dose responses has long been recognized, first being reported by the well known physiologist Rudolph Virchow in the mid $1850 \mathrm{~s}^{[2]}$. However, biphasic dose responses became very well known some thirty years later as the German physician and pharmacologist Hugo Schulz, not only repeatedly observed such dose responses in various biological systems $^{[3,4,5]}$ but also sought to generalize his observations in such a manner as to claim that it provided the scientific foundations of the medical practice of homeopathy. In fact, the entire subsequent professional career of Hugo Schulz which spanned some five decades, was committed to this prospective, making him and his theory highly controversial. Unfortunately, it also made the concept of biphasic dose responses controversial and often the object of disrepute and skepticism ${ }^{[6,7]}$. While disputes over whether biphasic dose responses were reproducible had their scientific foundations, they were often political as traditional medicine engaged in an intense and multifaceted battle with homeopathy over the future of medical treatment. In such high stakes battles Schulz and his biphasic dose response model did not fare well as they often became objects of ridicule and scientific misrepresentation and thus became collateral damage in a very protracted conflict that was finally decided in favor of traditional medicine ${ }^{[8]}$. While this battle was waged on multiple levels it also involved the scientific community as leading biomedical scientists, principally leading pharmacologists, used their knowledge and influence to discredit Schulz and his theories, leading to the creation of different dose response theories that became dominant in the early decades of the $20^{\text {th }}$ century and remain so today.

That current basic beliefs on the nature of the dose response arose out of an historical conflict between traditional medicine and homeopathy is an unrecognized and yet central feature that has significantly affected the course of modern toxicological and pharmacological sciences and the expansion of these concepts into the broader realm of the life sciences. The implications of the triumph of traditional medicine over homeopathy on the current state of beliefs on the nature of the dose response have been profound. By rejecting the biphasic dose response of Schulz, pharmacologists of the early part of the $20^{\text {th }}$ century were forced to propose an alternative dose response theory, the threshold model, along with statistical modeling ${ }^{[9]}$ and study designs to support it. The scientific leadership then built hazard assessment protocols around it for the testing of all chemicals and drugs. These ideas came to infiltrate all leading textbooks, professional societies, governmental regulatory agencies, testing protocols and exposure standards. In the end, the biphasic dose response became highly marginalized, whereas the threshold dose response model became that upon which modern society was built. The historical foundations of this unfolding have been presented in detail by Calabrese and Baldwin ${ }^{[10-14,8]}$.

The implications of the establishment of the threshold dose response model on the biomedical community is profound. In the first place, a belief in the threshold dose response model indicates an assumption that no treatment effect will occur below the threshold. This led to hazard assessment protocols being principally designed to assess responses that would estimate threshold responses and responses above the threshold. Hazard assessment protocols would not consider the likelihood or even the possibility that a real biological treatment effect would occur below the threshold. This assumption lead to toxicology being a high dose testing discipline, requiring only a few doses to achieve its testing goals. This intellectual framework was incorporated into the testing of the US FDA in the 1930s, later into the US PHS, subsequently into the National Cancer Institute carcinogen testing protocols and EPA and National Toxicology Progam (NTP) testing schemes. Other countries have followed these examples, building their testing and evaluative frameworks upon the belief that the most basic dose response model is the threshold.

Despite this near total domination of the threshold model within the biomedical community, biphasic dose responses continued to be published in the peer reviewed literature throughout the $20^{\text {th }}$ century and even more so in the first decade of the 21th century. In the early decades of the $20^{\text {th }}$ century such biphasic dose responses were commonly referred to as examples of the Arndt-Schulz Law ${ }^{[5]}$ or Hueppe's Rule ${ }^{[15]}$ but with most being referred to as a low dose stimulation and a high dose inhibition ${ }^{[16,17]}$. In $1943^{[18]}$ Southam and Ehrlich introduced the term hormesis into the biological lexicon based on their findings that low doses of extracts from the red cedar tree stimulated metabolism in various fungal species, while being inhibitory at higher doses. The term hormesis had a slow start with respect to recognition and use. In fact, the first time it is used in the Web of Science database is 1945, with the second entry being some 15 years later in 1960. Table 1 shows the time frame over which the term hormesis was cited in the Web of Science. This information indicates that the term hormesis started to become cited 
Am. J. Pharm. \& Toxicol., 3 (1): 59-71, 2008

Table 1: Frequency of citation by 10-year period in Web of Science of terms that could describe hormesis 1945-2007

\begin{tabular}{llllllll}
\hline & \multicolumn{2}{l}{ Frequency of Citation } & & & \\
Term & $1945-1954$ & $1955-1964$ & $1965-1974$ & $1975-1984$ & $1985-1994$ & $1995-2004$ & $2005-2007$ (Oct. 13) \\
\hline Bell-Shaped Curve & 0 & 0 & 0 & 1 & 193 & 495 & 119 \\
Bell-Shaped Dose-Response & 0 & 0 & 0 & 2 & 97 & 252 & 41 \\
U-Shaped Dose-Response & 0 & 0 & 0 & 0 & 48 & 195 & 55 \\
U-Shaped Curve & 0 & 0 & 0 & 1 & 149 & 408 & 136 \\
J-Shaped Curve & 0 & 0 & 0 & 0 & 21 & 114 & 34 \\
Biphasic Dose-Response & 0 & 0 & 1 & 9 & 182 & 346 & 62 \\
Functional Antagonism & 0 & 2 & 0 & 23 & 341 & 1,235 & 304 \\
Hormesis & 1 & 1 & 0 & 10 & 92 & 485 & 229 \\
\hline
\end{tabular}

in the late 1970s, with many more citations occurring from the late 1980 s to the present. Nearly $80 \%$ of the citations on hormesis have been in the first seven years of the present decade. Interestingly, the rather late citations of hormesis are similarly reflected with the temporal pattern of citations of the other alternative terms (Table 1) (e.g., U-shaped, J-shaped, biphasic, etc.). These findings indicate that the widespread recognition of biphasic dose responses has been of a relatively recent nature.

The recognition of biphasic dose responses has involved therefore similarly enhanced temporal citations in different disciplines: 1) Flood $^{[19],}$ building on a spate of reports from the University of CaliforniaBerkeley, Department of Psychology in the late 1960's ${ }^{[20]}$, galvanized this research concerning Ushaped dose responses on memory acquisition which has continued to the present. (2) Szabaldi ${ }^{[21]}$, researching in the area of pharmacology, developed his biphasic dose-response receptor-based mechanism interpretation. (3) Stebbing reported on the occurrence of similar biphasic dose response relationships in the late 1970s and early 1980s concerning the effects of water contamination on marine microorganisms within the context of homeostatic feedback regulatory mechanisms. (4) Luckey, in the late 1970s/early 1980s, comprehensively documented the occurrence of hormesis induced by low levels of radiation on biological models from plants to microorganisms to humans. (5) The field of epidemiology was also beginning to report U-shaped dose responses for various types of diseases, including the now well established relationships between alcohol consumption and cardiovascular disease ${ }^{[22]}$. (6) The adaptive response for mutational agents was reported in $1977^{[23]}$ and named a year later ${ }^{[24]}$. (7) Nearly a decade later the concept of a pre-conditioning to hypoxic stress was shown to provide protection against damage from the induction of myocardial infarction ${ }^{[25]}$. In both of these cases, the adapting and conditioning doses display inverted $U$ shaped dose responses and conform to the quantitative features of the hormetic dose response ${ }^{[26,27]}$.
It is very likely that these later two phenomena are manifestations of special cases of the hormetic dose response relationship.

\section{QUANTITATIVE FEATURES OF HORMETIC DOSE RESPONSES}

Despite the temporal convergence of biphasic dose response relationships there has been little interdisciplinary intellectual integration, with most disciplines seeing their own biphasic dose response as apparently unique, not part of a more generalized pattern of biological responses. This is reflected in the use of the broad range of terms given above for the discipline specific biphasic dose responses. However, in 1997 Calabrese and Baldwin ${ }^{[28]}$ reported on the creation of a hormetic dose response database that assimilated dose responses on the basis of various parameters including their study designs, dose response features, statistical analyses and reproducibility. Based on an analysis of this expanding database Calabrese and Blain $^{[1]}$ reported that the vast majority of biphasic dose responses in the biological literature display remarkably similar quantitative features as measured by the maximum stimulatory response, the width of the stimulation and the relationship of the stimulatory response to the toxicological/pharmacological threshold. More specifically, the maximum stimulatory response was generally modest with the strong majority of examples being only $30-60 \%$ greater than the controls (Fig. 3). The width of the stimulatory response was more variable, but was generally less than 100-fold as measured by the distance from the threshold or zero equivalent point (Fig. 4). On occasion very broad stimulatory ranges were reliably reported that extended beyond 1000-fold. The causes of the broad stimulatory ranges are generally unknown but most likely as related, as least in part, to the heterogeneity of the study population. Figure 5 provides a range of graphs that illustrate representative examples of hormetic dose response relationships. 
Am. J. Pharm. \& Toxicol., 3 (1): 59-71, 2008

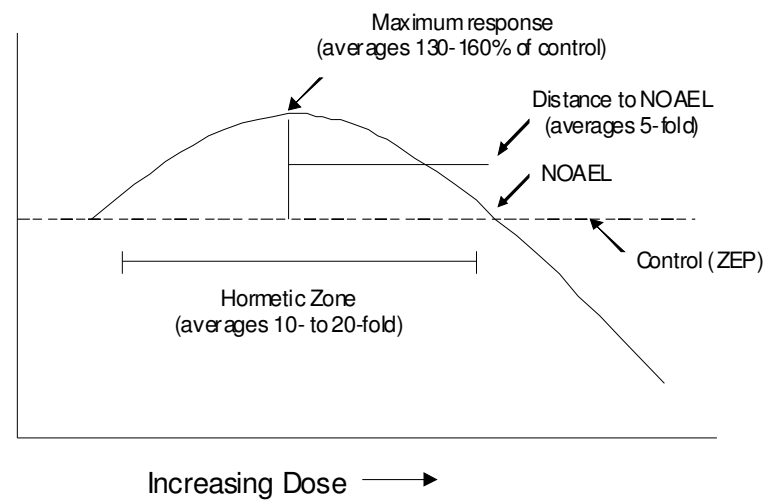

Fig. 3: Dose-response curve depicting the quantitative feature of hormesis

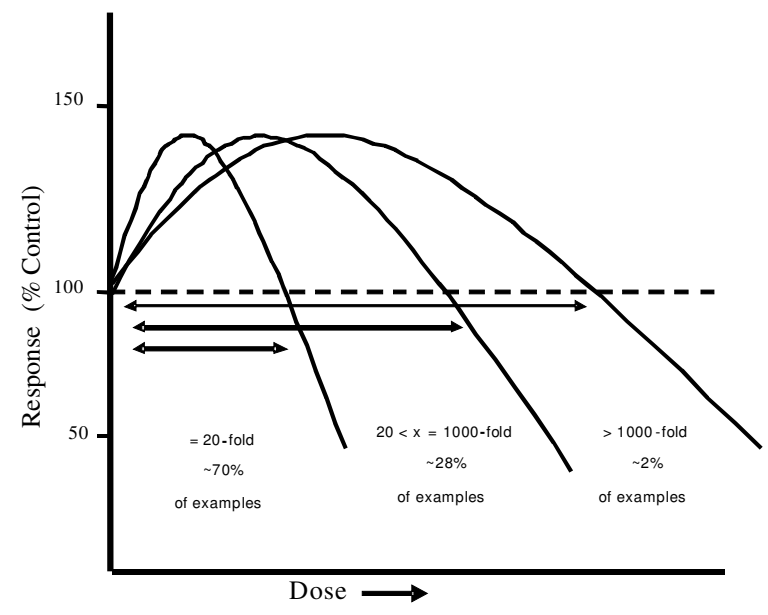

Fig. 4: Stylized dose-response curves reflecting the relative distribution of stimulatory dose ranges. Note: the maximum stimulatory response is usually $130-160 \%$ of the control value

The findings that have emerged from an assessment of the hormesis database suggest that the biphasic dose responses that are now widely reported in numerous biomedical disciplines display strikingly similar quantitative patterns. This is the case independent of the biological model, the endpoint measured and the chemical class or physical agent studied. This is also the case whether the findings are based on data derived from in vitro or in vivo studies, as well as whether the endpoints are cell specific, organ specific or an integrative organismal response such as growth or longevity. This similar quantitative response strategy is seen as an important finding that has been previously either missed or largely ignored within the various biomedical domains.

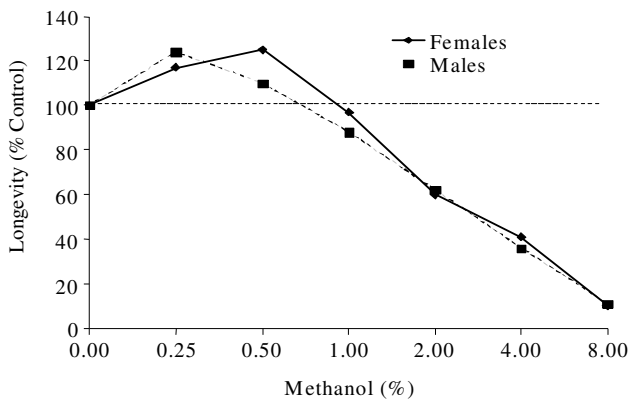

Fig. 5 (a): Methanol and fruit fly longevity ${ }^{[39]}$

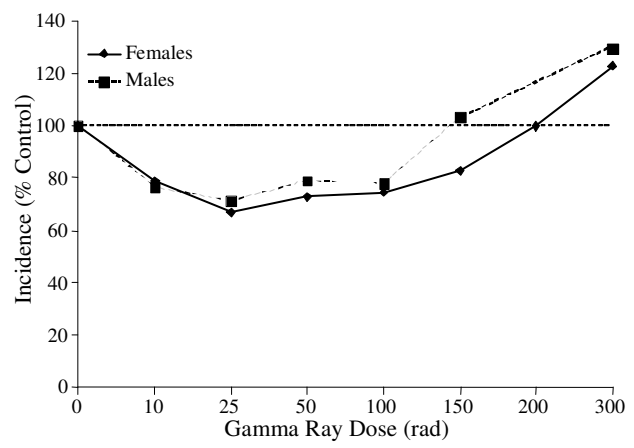

Fig. 5 (b): Gamma rays and mouse lung adenomas ${ }^{[40]}$

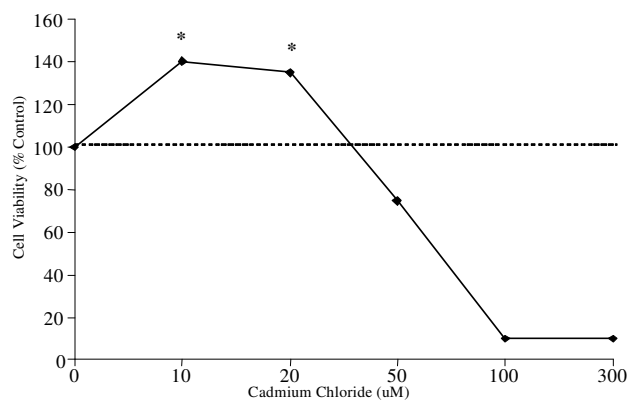

Fig. 5 (c): Cadmium and human ovarian cancer cell line viability $^{[41]}$

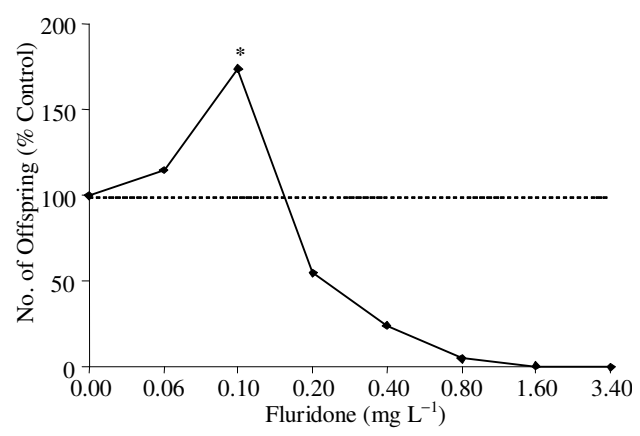

Fig. 5 (d): Fluridone and daphind fecundity ${ }^{[42]}$ 
Am. J. Pharm. \& Toxicol., 3 (1): 59-71, 2008

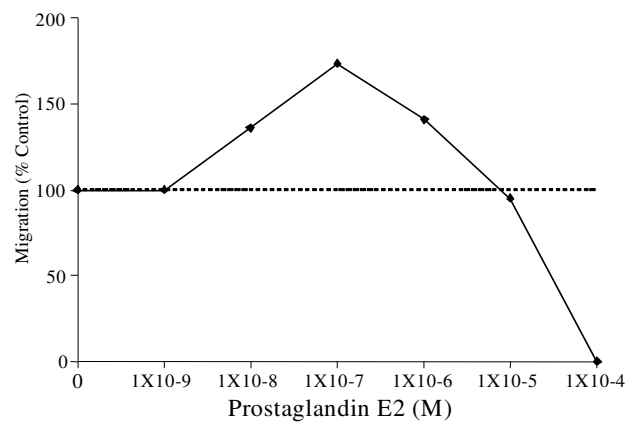

Fig. 5 (e): Prostaglandin E2 and human polymorphonuclear leukocyte chemotaxis ${ }^{[43]}$

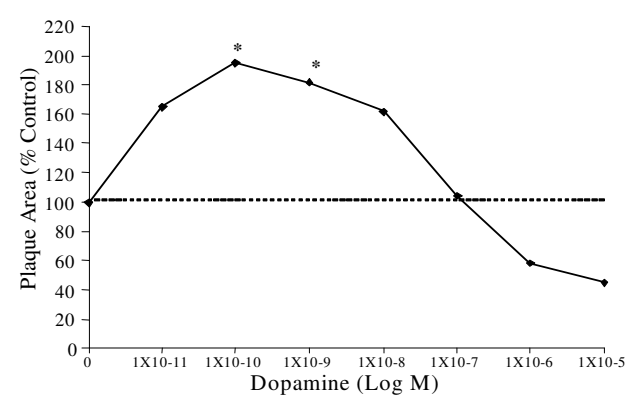

Fig. 5 (f): Dopamine and rat pituitary cell prolactin secretion $^{[44]}$

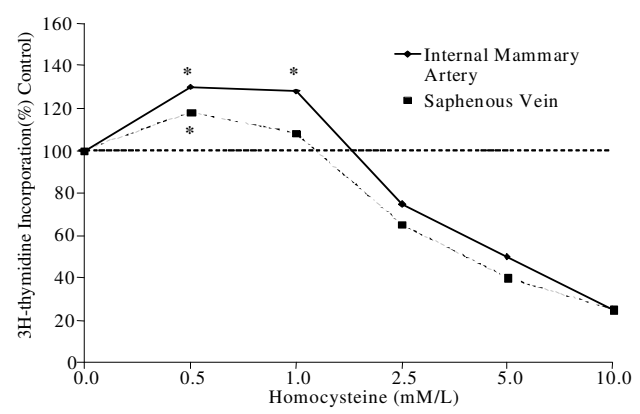

Fig. 5 (g): Homocysteine and human vascular cell growth $^{[45]}$

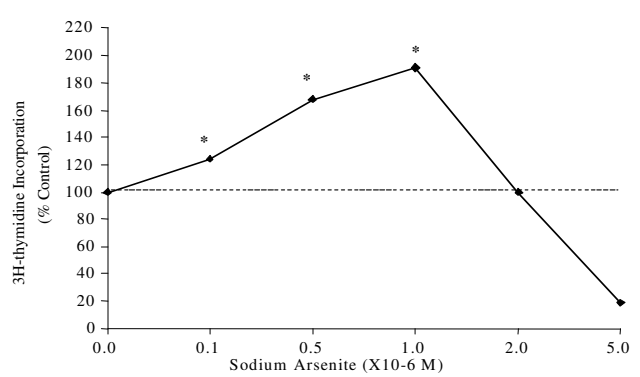

Fig. 5 (h): Arsenic and human lymphocyte DNA synthesis $^{[46]}$

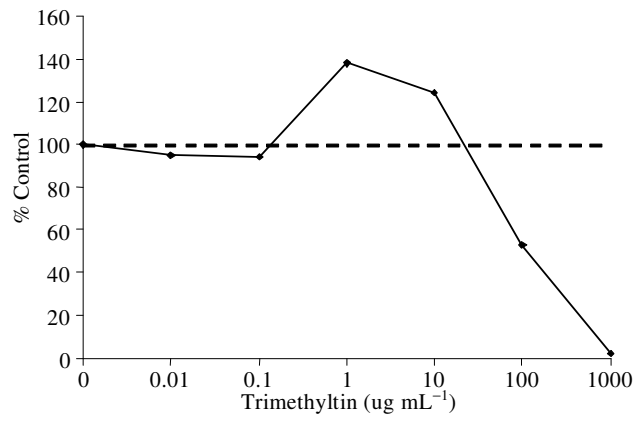

Fig. 5 (i): Effect on primary astrocyte cultures with the MTT assay ${ }^{[47]}$

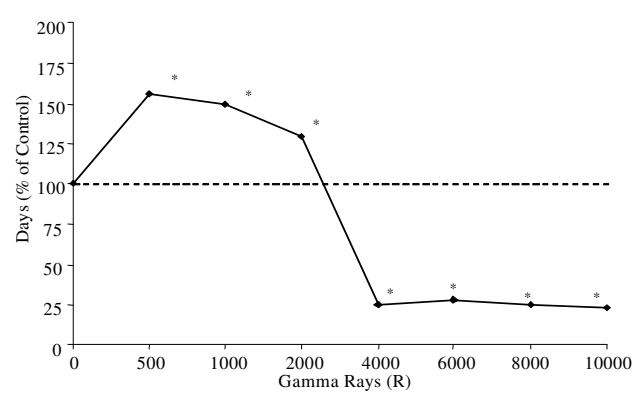

Fig. 5 (j): Effect of gamma rays on the life span of female house cricket ${ }^{[48]}$

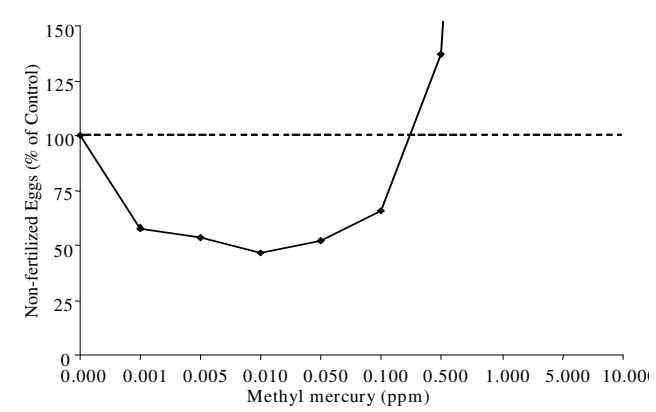

Fig. 5 (k): Effect of methyl mercury on the percent of non-fertilized eggs in steelhead trout ${ }^{[49]}$

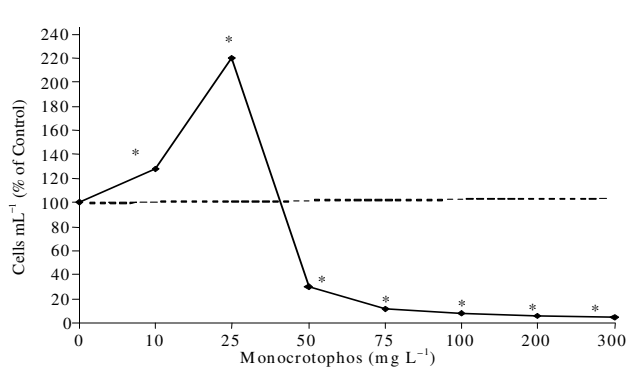

Fig. 5 (1): Effect of monocrotoph on the cell number of cultured algae $^{[50]}$ 
Am. J. Pharm. \& Toxicol., 3 (1): 59-71, 2008

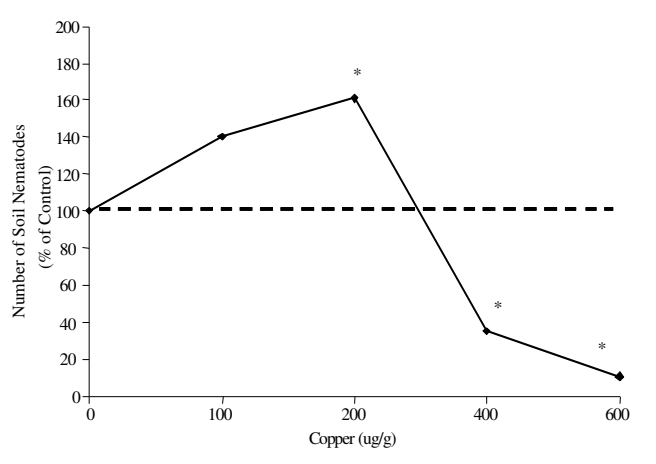

Fig. $5(\mathrm{~m})$ : Effect of copper on the population growth of nematodes ${ }^{[51]}$

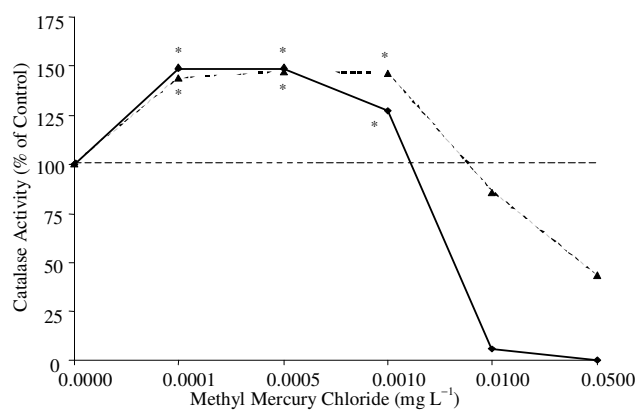

Fig. 5 (n): Effect of methyl mercury chloride on catalase and peroxidase activity in duckweed $^{[52]}$

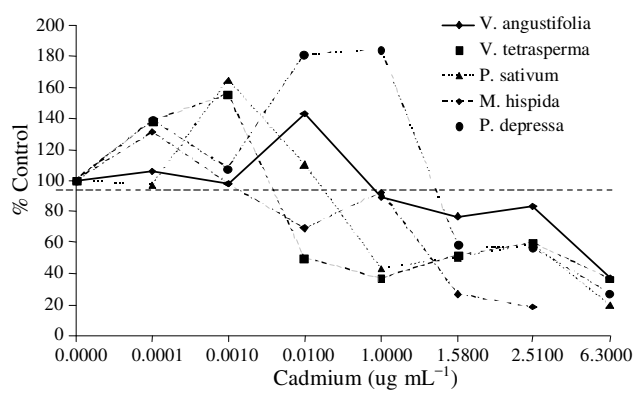

Fig. 5 (o): Pollen tube lengths of five species exposed in vitro to cadmium ${ }^{[53]}$

The underlying reasons to account for the quantitative features of the hormetic dose response relationship remain speculative. However, given the widespread generalizability of the quantitative features of the dose response it suggests that they may be a measure of plasticity in biological systems across various levels of organizations. However, the constraints on plasticity must take into account efficiencies of resource management and allocation as well as resources required during the process of tissue repair and a wide range of other adaptive processes.

\section{MECHANISMS}

There is a general, but mistaken, assumption that there is little mechanistic understanding of hormetic dose response relationships. Placed in perspective, it should be noted that in the field of toxicology, there has been little attention and effort directed to assessing mechanisms that account for dose dependent changes in the shape of the dose response. However, some research groups have begun to make contributions in this area. For example, Japanese researchers have explored underlying mechanisms that may account for the consistent pattern of $\mathrm{J}$-shaped dose responses for liver tumors and foci induced by non-genotoxic carcinogens $^{[29]}$. For example, in the case of DDT these investigators have demonstrated that pro-oxidant mechanisms dominate the cellular environment within the high dose range. In contrast, in the area of the reduced tumor/foci incidence various types of adaptive responses are upregulated that are believed to have contributed to the protective response. A plethora of mechanisms have been proposed to account for the occurrence of neuroprotective effects reported in a variety of neuronal cell types $^{[30]}$.

In the area of pharmacologically based direct stimulation hormesis, Szabaldi ${ }^{[21]}$ initially proposed that such responses could be readily accounted for by the existence of one agonist interacting with two receptor subtypes whose activation would lead to pathways of stimulation and inhibition, respectively. In this situation there would be differential affinities for the two receptor subtypes, with the receptor with the greatest affinity having lower capacity (i.e., fewer receptors) receptors and the reverse. At low concentrations the receptor subtype with the greatest affinity would dominate the dose response relationship. However, as the concentration increases, the receptor subtype with the lower affinity but with the greater capacity, that is, more receptors, would come to dominate the dose response at the higher doses. When integrated and expressed across the entire dose response continuum, a biphasic dose response becomes evident. This type of receptor based mechanism has been reported in nearly 30 different receptor systems accounting for the observed biphasic dose response (Table 2). Despite this mechanistic accounting for the shape of the dose response it has not yet been applied to explain the basis for the maximum stimulatory response, nor the width of the stimulation. 
Am. J. Pharm. \& Toxicol., 3 (1): 59-71, 2008

Table 2: Representative receptor systems displaying biphasic doseresponse relationships

\begin{tabular}{ll}
\hline Adenosine & Neuropeptides \\
\hline Adrenergic & Nitric Oxide \\
Bradykinin & N-Methyl-D-Aspartate \\
Cholecystotkinin & Opioid \\
Corticosterone & Platelet-Derived Growth Factor \\
Dopamine & Prolactin \\
Endothelin & Prostaglandin \\
Epidermal Growth Factor & Somatostatin \\
Estrogen & Spermine \\
5-Hydrosytryptamine (Serotonin) & Testosterone \\
Human Chorionic Gonadotrophin & Transforming Growth Factor $\beta$ \\
Muscarinic & Tumor Necrosis Factor $\alpha$ \\
\hline
\end{tabular}

\section{STUDYING HORMESIS}

In order to assess hormesis it is necessary to have robust study designs, with sufficient numbers of doses, proper dose spacing and adequate statistical power. In practice, this may require the estimation of the toxicological/pharmacological threshold with preliminary testing. Such data would enhance the likelihood for experiments to include doses both above and below the estimated threshold. This is important since it would provide data on the broad features of the dose response relationship. The number of doses below the threshold and their spacing can follow some guidance obtained from a review of thousands of examples of hormetic dose response relationships ${ }^{[1]}$; it also needs to be tempered by knowledge of the specific biological model and experimental conditions. In general, there should be at least two doses above the threshold response to help ensure that dose responses would display clear and highly confident inhibitory trends consistent with a traditional dose-response relationship, it is especially helpful to have at least three doses below the threshold in order to assess hormetic responses, although there often has been less. Based on considerable evidence it is expected that the hormetic response should become evident within about 1/3-1/5 of the estimated threshold dose. The maximum stimulatory response would likely occur within $1 / 5$ $1 / 20^{\text {th }}$ of the threshold response. It is not certain how broad the stimulatory range may be. If there were a third dose it could be in the $1 / 100-1 / 20^{\text {th }}$ range of the threshold dose. It would be of value, of course, to have sufficient doses to properly define the entire dose response continuum. However, there is always going to be the issue of resources and time constraints.

The assessment of hormesis has been markedly enhanced with the onset of cell culture experimentation since the mid 1980s since they require fewer resources, less time and permit more doses to be assessed. For example, there have been numerous experiments with
96 well plates in which 10 or 11 concentrations are tested along with controls and eight replications on the same plate. In such cases the use of large numbers of concentrations permits the assessment of the above threshold and below threshold concentrations in a manner that facilitates an evaluation of hormetic dose response hypotheses.

It is also important not to neglect the issue of temporal factors affecting the understanding of the dose response relationship ${ }^{[31]}$. A key concept is that a biological system's response to the stress or damage is critical to evaluate. This requires the careful assessment of toxic and reparative changes over time. This has been demonstrated to be a key factor in essentially all biological systems, yet it is often overlooked.

Of particular importance in the design of experiments dealing with hormesis is that the expected maximum response would only be about $30-60 \%$ greater than control values. This may create study design concerns with respect to statistical power issues. Study designs could be unbalanced with higher numbers of subjects being included in those doses that are below the threshold since any possible stimulatory response is likely to be modest. This modest magnitude of the hormetic stimulatory response also increases the need to adequately replicate study findings.

The demands for stronger study designs, with more doses, higher numbers of subjects, the incorporation of temporal features and the greater need to replicate findings places special challenges on those interested in assessing hormetic dose responses. While this can discourage investigators from exploring hormesis, this is likely to become more common as low dose phenomena become more significant. There is no easy solution to the resource and time issue, with the exceptions, as noted above, of cell culture experimentation. Likewise, there is also the possibility of using less expensive biological models such as plants, algae and various micro-organisms.

Major governmental hazard assessment programs which often employ only three doses, with an emphasis on estimating the NOAEL and toxic doses above this value fail to address the possibility that hormetic effects may occur. This few doses-high dose strategy is in direct conflict with the intention of estimating biological changes in the low dose zone, that is, below the threshold response.

Another important concern when assessing hormetic effects is the control group background disease or response incidence. It is practically impossible to assess an hormetic hypothesis if the disease incidence is very low to negligible. Yet, from an historical perspective the selection of animal models 
for the U.S. cancer bioassay program was based principally on the models not being very susceptible to infectious disease and having a low background disease incidence for the endpoints of interest. This latter feature was driven by a desire to maximize statistical power with the lowest possible number of animals. While this is a valid concern, it conflicts with the capacity to assess possible hormetic dose responses.

A different concern in assessing hormetic effects can also arise when control groups display very low or negligible background values. The issue is not assessing whether the disease incidence can be decreased as in the example above, but rather the opposite. That is, it would involve parameters that would be expected to increase following treatment. In this instance the background may be so low that even modest responses may represent increases of many hundreds fold. However, it appears that hormetic responses have been typically studied when the background has achieved a steady state that is appreciably greater than negligible. This is likely to become an area of greater discussion in the future, as gene products become more routinely measured.

\section{FINDING EXAMPLES OF HORMESIS}

The easiest approach to finding examples of hormesis would seem to start with a search of various electronic databases. For example, a search of PubMed toward the end of 2007 indicated about 450 articles whereas a similar search using the Web of Science yielded about 850 articles. However, based on our research in the development of the hormesis database ${ }^{[1]}$, it is clear that reliance of these databases and other standard electronic database offerings will underestimate the occurrence of hormesis by approximately 10-20-fold! One could improve their search capacity further by using other terms for hormetic dose responses as noted above. However, in our experience the majority of articles with evidence on hormesis will not be identifiable even by use of all of the above terms and by cross referencing. This is because investigators often do not discuss the low dose stimulatory response that may be reported in their figures or tables. In such situations, these responses are usually discovered by casual observation or by deliberate time consuming efforts to scan large numbers of articles in journals likely to contain a relatively high proportion of dose response relationships. Thus, finding hormetic dose response relationships in the biomedical literature is not an easy or efficient task. Yet, it is important to understand the limitations of current search strategies and the extra effort required to discover hormetic dose responses.

\section{BIOMEDICAL IMPLICATIONS}

Low doses of anti-tumor agents: Low doses of antitumor agents often stimulate the proliferation of tumor cells following the quantitative features of the hormetic dose response. This has been shown for numerous human tumor cell lines and a wide diversity of chemical agents. In some cases investigators have become concerned that low doses of these agents may present risks to patients. Such potential risks would be further enhanced if the agent had a long biological half-life in the patient, for example suramin, which acts in such an hormetic fashion, has a half life in humans of over one month $^{[32]}$. Further, Calabrese et al. ${ }^{[33]}$ have demonstrated that a sizable proportion of possible antitumor agents tested in NCI anti-tumor yeast screening bioassays also stimulate cell proliferation at low doses. These findings suggest that hormetic effects need to be taken into account in the design of new anti-tumor drug treatments, in the selection of drugs for patient treatment and in the measurement of drug concentrations following treatment. It would be ideal to use agents that did not stimulate the proliferation of tumor cells at low doses. However, if this situation can not be avoided then it would be important to select drugs with very short half-lives. This problem with anti-tumor drugs is also likely to be the case with antibacterial, antifungal and antiviral agents as well.

Expected performance enhancements based on hormesis: The quantitative features of the hormetic dose response indicates that the typical maximum response is in the $30-60 \%$ range. In the analysis of thousands of examples of hormetic dose response relationships, this is the most striking and consistent finding. It suggests that drug induced performance increases will be constrained by this limitation in biological plasticity. This is the case whether the goal is to enhance memory, grow hair, increase seizure thresholds, reduce anxiety, modulate pain, protect neurons, enhance neuronal growth, increase plant productivity and other biomedical or commercial goals. The constraints imposed by the quantitative features of the hormetic dose response need to be taken into consideration throughout the entire process of drug development and evaluation.

Hormesis and pharmaceuticals: Even though it is not generally appreciated, pharmaceutical agents routinely display hormetic dose responses and, in fact, have been 
Am. J. Pharm. \& Toxicol., 3 (1): 59-71, 2008

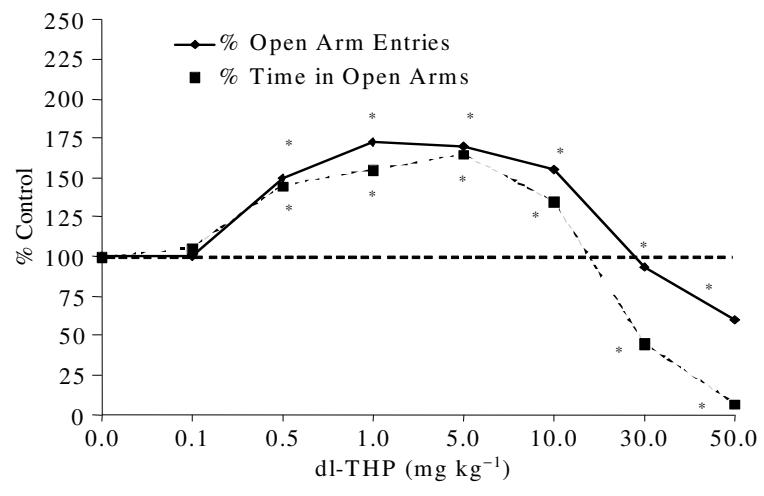

Fig. 6: Anxiolytic effect of dl-THP on male and female ICR mice in the elevated plus-maze test. *Significantly different from controls at $\mathrm{p}<0.05$. (Source: Leung et al., 2003, Fig. 2)

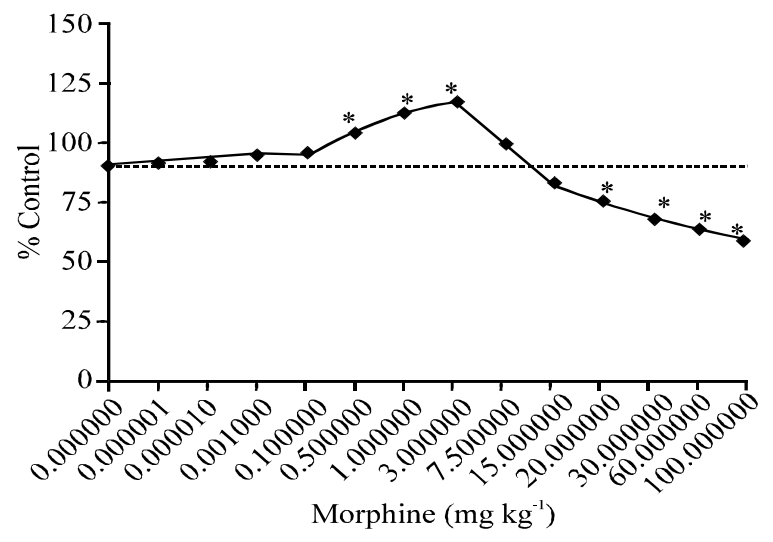

Fig. 7: Effect of different doses of morphine on PTZinducted seizure threshold. *Significantly different from controls at $\mathrm{p}<0.05$. (Source: Honar et al., 2004, Fig. 1).

selected because of it. For example, the capacity of anxiolytic drugs to reduce anxiety in animal models typically do so in a manner fully consistent with the hormetic dose response ${ }^{[34]}$ (Fig. 6). That is, the low dose stimulation is a measure of the reduction in anxiety. This is also the case with anti-seizure drugs that are capable of increasing the seizure thresholds in predictive animal models ${ }^{[35]}$ (Fig. 7). The dose response features of these agents reflect the quantitative features of the hormetic dose response with respect to the maximum stimulatory response, the width of the stimulatory zone and the relationship of the stimulatory response to the threshold. Thus, the hormetic dose response should be considered an important element, informing preclinical testing programs and the design and conduct of clinical trials.
Multiple chemical interactions and synergistic responses: In the domain of toxicology chemical interactions explore whether agents may act in additive, synergistic or antagonistic fashions. Even though they are not common, striking synergistic effects have been reported in which toxicities may be profoundly enhanced. For example, the interaction between very low (i.e., non-toxic) doses of the banned pesticide kepone and carbon tetrachloride rapidly kill adult Sprague-Dawley rats ${ }^{[36]}$. The concept of chemical interactions is different for hormetic dose responses than seen within the context of a toxic response. In the case of hormesis the issue is how much performance, rather than toxicity, will be affected. In studies dealing with memory enhancement it was determined that performance could not be enhanced beyond the 30-60\% range regardless of how the dosing schemes were arranged $^{[20]}$ even in situations in which powerful synergistic interactions occurred. It therefore appears that the interactions occur within a response zone that is highly constrained. This performance based synergistic concept is fundamentally different than that addressed in toxicological settings. The implications are important because the interaction is more about achieving a restricted maximal response with very small drug combinations rather than profoundly increasing the response.

Flawed dose-response models used by regulatory agencies: There are thousands of examples of hormesis obtained from the peer-reviewed literature which have been assessed and entered into the hormesis database. On the basis of these observations alone, one may infer that the widely used dose response models such as the threshold and linear at low doses have important limitations concerning generalizability, raising doubts as to the scientific foundations upon which they have been selected as default models in risk assessment procedures used by federal and state agencies throughout the United States and other countries. However, even more significant is that in head to head competition using identical a priori entry and evaluative criteria, the hormetic dose response model has been far superior to its rival models, such as the threshold model, whether dealing with environmental chemicals $^{[37,38]}$ or large numbers of anti-tumor agents tested by the US National Cancer Institute ${ }^{[33]}$ in predicting the responses of agents below the toxic threshold, that is, in the low dose zone. These studies were particularly valuable, not just for the fact that hormesis yielded accurate predictions, but for exposing the serious limitations of the threshold dose response model to accurately predict below threshold responses. 
Since humans are expected to be exposed to agents at doses below the toxic threshold, risk assessment models should have a good to excellent capacity to predict responses below the threshold in a reliable manner. This is not something that the threshold model has been able to do when tested. Given its consistent history of failure it is questionable whether it will perform markedly better when assessing other biological systems and agents. Even if it did, the question would arise as to why it would be so inconsistent in its performance. These findings seriously question the predictive capacity of currently used default dose response models; it also raises the question that objective criteria should be established by which default dose response models should be selected.

\section{RESULTS AND DISCUSSION}

This research has revealed that hormesis is a fundamental dose-response strategy of biological systems across multiple aspects of biological organizations, from the cell to populations. Based on this assessment a series of Hormetic Principles have emerged that provide an improved articulation of its biological significance (Table 3). These principles are themselves derived from an analysis of the general findings of thousands of articles that have assessed hormetic hypotheses, thereby revealing its biological features and limitations (Table 4). The derivation of general hormetic principles based on substantial data provides a solid framework to explore its scientific implications, some of which are noted in Table 5.

Table 3: Hormetic Principles

- Hormesis is a case of "biological leveraging" in which a stress or slight damage is experienced in the expectation that it will induce a compensatory response sufficient to produce a net benefit that more than covers the biological costs of the initial stress.

- $\quad$ Low/modest stress observed in hormetic dose-responses induces pro-survival responses.

- Hormesis dose responses (HDR) may be seen as an adaptive response that ensures tissue repair in an efficient manner and protects against damage from subsequent and more massive exposures.

- The quantitative features of the hormetic dose response are similar across species and individuals and independent of differential susceptibility, endpoint measured and differential agent potency.

- The magnitude of the hormetic stimulatory response is constrained by and defines the plasticity of the biological system.

- Hormetic responses occur at multiple levels of biological organization, the cellular, organ, individual and population.

- Downstream processes integrate responses from multiple independent stressor agents/excitatory stimuli to yield an integrated dose response (i.e., molecular vector) reflecting the hormetic dose response.

- Hormetic responses reflect both a general response to environmental induced stress/damage as well as elements of chemical structure specificity for endpoint induction.

Table 4: Major hormetic dose response observations

- Hormetic dose-response relationships are the most commonly observed dose response relationship in standardized testing.

- Hormetic dose-response relationships display distinctive quantitative features, making it a specific type of biphasic dose response relationship.

- Most unique feature of the hormetic dose response is the modest magnitude of the stimulatory response, usually less than twice control values.

- The low dose hormetic stimulation can occur via a direct stimulation or via an overcompensation to a disruption of homeostasis.

- $\quad$ Numerous specific mechanisms have been reported to account for hormetic dose responses.

- Hormetic synergy occurs within the constraints placed on the quantitative features of the dose response; thus, synergy is seen less on the effect than in the dose to achieve the "constrained" synergistic effect.

Table 5: Implications of hormesis for toxicological/clinical practices

Toxicology/Risk Assessment

- Changes strategy for hazard assessment, altering animal model and endpoint selection, study design, including number of doses, and dose range and number of subjects per dose.

- Alters biostatistical modeling to predict estimates of response below control background disease incidence.

- Differentiates dose optima (i.e., benefits) for normal and high risk segments of the population.

- $\quad$ Creates evaluative framework to assess benefits or harm below traditional toxicological threshold.

- Creates new framework for quantitatively altering the magnitude of uncertainty factors in the risk assessment process.

Clinical Practices/Pharmaceutical Companies

- $\quad$ Drug performance expectation will be constrained by the quantitative features of the hormetic dose response.

- Drugs that are designed to act at high doses may have hormetic effects at low doses with possible undesirable effects (e.g., tumor cell proliferation).

- Modification of biological set points will be constrained by the quantitative features of the hormetic dose response.

- Clinical trials need to recognize inter-individual variation in the hormetic dose response.

- Clinical trials need to be designed to take into account the quantitative features of the hormetic dose response.

- Drugs may have multiple concurrent hormetic effects on different organ systems that have the potential to create a broad spectrum of beneficial and adverse effects.

- Partial agonist-antagonists often induce U-shaped dose response with fewer side effects and with broader therapeutic zone than full agonists. This not only suggests their practical use in clinical pharmacology but also provides an explanation for partial agonist-antagonists induced U-shape dose responses within a natural selection framework. 
Hormesis is expected to become an ever more appreciated dose-response concept that will lead to markedly improved efforts in drug discovery and development processes as well as improving the process of hazard assessment for drugs and environmental chemicals and providing critical new insights in the process of cost-benefit analysis for chemical and pharmaceutical agents.

\section{ACKNOWLEDGMENT}

Effort sponsored by the Air Force Office of Scientific Research, Air Force Material Command, USAF, under grant number FA9550-07-1-0248. The U.S. Government is authorized to reproduce and distribute for governmental purposes notwithstanding any copyright notation thereon. The views and conclusions contained herein are those of the authors and should not be interpreted as necessarily representing the official policies or endorsement, either expressed or implied, of the Air Force Office of Scientific Research or the U.S. Government.

\section{REFERENCES}

1. Calabrese, E.J., R. Blain, 2005. The occurrence of hormetic dose responses in the toxicological literature, the hormesis database: An overview. Toxicol. Appl. Pharmacol., 202: 289-301.

2. Henschler, D., 2006. The origin of hormesis: Historical background and driving forces. Hum. Exp. Toxicol., 25: 347-351.

3. Schulz, H., 1887. Zur Lehre von der Arzneiwirdung. Virchows Archiv fur Pathologische Anatomie und Physiologie fur Klinishce Medizin 108:423-445.

4. Schulz, H., 1888. Uber Hefegifte. Pflugers Archiv fur die gesamte Physiologie des Menschen und der Tiere 42: 517-541.

5. Crump, T., 2003. NIH Library Translation (NIH98-134). Contemporary Medicine as Presented by its Practitioners Themselves, Leipzig, 1923:217250, Hugo Schulz. Nonlin. Biol. Toxicol. Med., 1: 295-318.

6. Clark, A.J., 1937. Handbook of Experimental Pharmacology. Verlig Von Julius Springer, Berlin.

7. Clark, A.J., 1927. The historical aspects of quackery, Part 2. Brit. Med. J., 1927: 960-960.

8. Calabrese, E.J. , 2005a. Historical blunders: How toxicology got the dose-response relationship half right. Cell Mol. Biol., 51: 643-654.

9. Bliss, C.I., 1935. The comparison of dosagemortality data. Ann. Appl. Biol., 22: 307-333.
10. Calabrese, E.J. and L.A. Baldwin, 2000a. Chemical hormesis: its historical foundations as a biological hypothesis. Hum. Exp. Toxicol., 19: 2-31.

11. Calabrese, E.J. and L.A. Baldwin, 2000b. The marginalization of hormesis. Hum. Exp. Toxicol., 19: 32-40.

12. Calabrese, E.J. and L.A. Baldwin, 2000c. Radiation hormesis: Its historical foundations as a biological hypothesis. Hum. Exp. Toxicol., 19: 41-75.

13. Calabrese, E.J. and L.A. Baldwin, 2000d. Radiation hormesis: The demise of a legitimate hypothesis. Hum. Exp. Toxicol., 19: 76-84.

14. Calabrese, E.J. and L.A. Baldwin, 2000e. Tales of Two Similar Hypotheses: The rise and fall of chemical and radiation hormesis. Hum. Exp. Toxicol., 19: 85-97.

15. Hueppe, F., 1896. Principles of Bacteriology. Translated from the German by E.O. Jordan. The Open Court Publishing Company. Chicago, IL.

16. Hotchkiss, M., 1923. Studies on salt action. VI. The stimulating and inhibitive effect of certain cations upon bacterial growth. J. Bact., 8:141-162.

17. Hotchkiss, M., 1923. The influence of various salts upon the growth of bacterium communis. P.hD Thesis, Yale University.

18. Southam, C.M. and J. Erhlich, 1943. Effects of extracts of western red-cedar heartwood on certain wood-decaying fungi in culture. Phytopathology, 33:517-524.

19. Flood, J.F., G.E. Smith and E. Roberts, 1988. Dehydroepiandrosterone and its sulfate enhance memory retention in mice. Brain Res., 447: 269-278.

20. Calabrese, E.J., 2008d. Alzheimer's disease drugs: An application of the hormetic dose response model. Crit. Rev. Toxicol., 38(5):(In press).

21. Szabadi, E., 1977. A model of two functionally antagonistic receptor populations activated by the same agonist. J. Theor. Biol., 69: 101-112.

22. Marmot, M.G., G. Rose, M.J. Shipley and B.J. Thomas, 1981. Alcohol and mortality-a U-shaped curve. Lancet, 1: 580-583.

23. Samson, L. and J. Cairns, 1977. A new pathway for DNA in Escherichia coli. Nature, 267:281-283.

24. Schendel, P.F., M. Defais, P. Jeggo, L. Samson and J. Cairns, 1978. Pathways of mutagenesis and repair in Escherichia-coli exposed to low-levels of simple alkylating-agents. J. Bacteriol., 135: 466-475.

25. Murry, C.E., R.B. Jennings and K.A. Reimer, 1986. Preconditioning with ischemia-a delay of lethal cell injury in ischemic myocardium. Circulation, 74: 1124-1136. 
26. Calabrese, E.J., 2008c. Converging concepts: Adaptive response, preconditioning and the Yerkes-Dodson Law are manifestations of hormesis. Ageing Res. Rev., 7:8-20.

27. Calabrese, E.J., et al. (more than 50 authors), 2007. Biological stress response terminology: Integrating the concepts of adaptive response and preconditioning stress within a hormetic doseresponse framework. Toxicol. Appl. Pharmacol., 222: $122-128$

28. Calabrese, E.J. and L.A. Baldwin, 1997. The dose determines the stimulation (and poison): Development of a chemical hormesis database. Int. J. Toxicol., 16:545-559.

29. Kinoshita, A., H. Wanibuchi, M. Wei and S. Fukushima, 2006. Hormesis in carcinogenicity of non-genotoxic carcinogens. J. Toxicol. Pathol., 19:111-122.

30. Mattson MP and A.W. Cheng, 2006. Neurohormetic phytochemicals: Low-dose toxins that induce adaptive neuronal stress responses. Trends Neurosci., 29: 632-639.

31. Calabrese, E. J., 2001. Overcompensation stimulation: a mechanism for hormetic effects. Crit. Rev. Toxicol., 31 (4): 425-470.

32. Calabrese, E.J., 2005b. Cancer biology and hormesis: Human tumor cell lines commonly display hormetic (biphasic) dose responses. Crit. Rev. Toxicol., 35: 463-582.

33. Calabrese, E.J., J.W. Staudenmayer, E.J. Stanek and G.R.Hoffmann, 2006. Hormesis outperforms threshold model in NCI anti-tumor drug screening data. Tox. Sci., 94: 368-378.

34. Calabrese, E.J., 2008a. An assessment of anxiolytic drug screening tests: Hormetic dose responses predominate. Crit. Rev. Toxicol., 38(6):(In Press).

35. Calabrese, E.J., 2008b. Modulation of the epileptic seizure threshold: Implications of biphasic dose responses. Crit. Rev. Toxicol., 38(6):(In Press).

36. Klingensmith, J.S. and H.M. Mehendale, 1982. Potentiation of $\mathrm{CCl} 4$ lethality by chlordecone. Toxicol. Lett., 11: 149-154.

37. Calabrese, E.J. and L.A. Baldwin, 2003. The hormetic dose response model is more common than the threshold model in toxicology. Toxicol. Sci., 71: 246-250.

38. Calabrese, E.J. and L.A. Baldwin, 2001. The frequency of U-shaped dose-responses in the toxicological literature. Tox. Sci., 62:330-338.

39. Jefferson, M.C. and M. Aguirre, 1980. Methanol tolerances and the effects of methanol on longevity and oviposition behavior in Drosophila pachea. Physiol. Entomol., 5: 265-269.
40. Ullrich, R.L. and J.B. Storer, 1979. Influence of yirradiation on the development of neoplastic disease in mice. Radiat. Res., 80: 317-324.

41. Abe, T., S. Gotoh and K. Higashi, 1999. Attenuation by glutathione of hsp72 gene expression induced by cadmium in cisplatinresistant human ovarian cancer cells. Biochem. Pharmacol., 58: 69-76.

42. Hamelink, J.L., 1986. Toxicity of fluridone to aquatic invertebrates and fish. Environ. Toxciol. Chem., 5:87-94.

43. Van Epps, D.E., A. Wiik, M.L. Garcia and R.C. Williams, 1978. Enhancement of human neutrophil migration by prostaglandin E2. Cell Immunol., 37: 142-150.

44. Burris, T.P., 1992. The stimulatory and inhibitory effects of dopamine on prolactin secretion involve different G-proteins. Endocrinology, 130:926-932.

45. Tang, L., C.D.S. Mamotte, F.M. Van Bockxmeer and R.R. Taylor, 1998. The effect of homocysteine on DNA synthesis in cultured human vascular smooth muscle. Atherosclerosis, 136: 169-173.

46. Meng, G., 1993. Effects of arsenic on DNA synthesis in human lymphocytes. Arch. Environ. Contam. Toxicol., 25: 525-528.

47. Cookson, M.R., C. Mead, S.M. Austwick and V.W. Pentreath, 1995. Use of the MTT assay for estimating toxicity in primary astrocyte and C6 glioma cell cultures. Toxicol. in vitro, 9:39-48.

48. Hunter, P.E. and V. Krithayakiern, 1971. Effect of gamma radiation upon life expectancy and reproduction in the house cricket, Acheta dome sticus (Orthopter:Fryllidae). Ann. Entomol. Soc. America, 64: 119-123.

49. McIntyre, J.D., 1973. Toxicity of methyl mercury for Steelhead trout sperm. Bull. Environ. Contam. Toxicol., 9: 98-99.

50. Nayak, S., R.C. Mohanty and L. Mohanty, 1996. Growth rate off ankistrodesmus falcatus and Scenedesmus bijuga in mixed culture exposed to monocrotophos. Bull. Enviorn. Contam. Toxicol., 57: 473-479.

51. Parmelee, R.W., R.S. Wentsel, C.T. Phillips, M. Simini and R.T. Checkai, 1993. Soil microcosm for testing the effects of chemical pollutants on soil fauna communities and trophic structure. Enviorn. Toxicol. Chem., 12: 1477-1486.

52. Subhara, A.V., A.K. Nanda, P.K. Behera and B.B. Panda, 1991. Acceleration of catalase and peroxidase activities in Lemna mino L. and Allium cepa L. in response to low levels of aquatic mercury. Environ. Pollut., 69: 169-179.

53. Xiong, Z-T. and Y-H. Peng, 2001. Response of pollen germination and tube growth to cadmium with special reference to low concentration exposure. Ecotoxicol. Enviorn. Safety, 48: 51-55. 